\title{
General strategy for the generation of human antibody variable domains with increased aggregation resistance
}

\author{
Kip Dudgeon ${ }^{\mathrm{a}, 1}$, Romain Rouet ${ }^{\mathrm{a}, \mathrm{b}, 1}$, Iris Kokmeijera, Peter Schofield ${ }^{\mathrm{a}}$, Jessica Stolp ${ }^{\mathrm{a}}$, David Langley ${ }^{\mathrm{a}, \mathrm{c}}$, \\ Daniela Stock ${ }^{\mathrm{b}, \mathrm{c}}$, and Daniel Christ ${ }^{\mathrm{a}, \mathrm{b}, 2}$
}

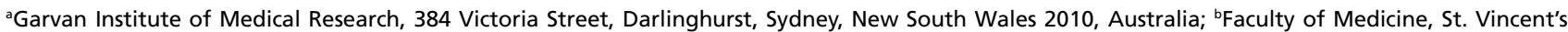
Clinical School, University of New South Wales, Darlinghurst, Sydney, New South Wales 2010, Australia; and 'Victor Chang Cardiac Research Institute, 405 Liverpool Street, Darlinghurst, Sydney, New South Wales 2010, Australia
\end{abstract}

Edited by Alan R. Fersht, MRC Laboratory of Molecular Biology, Cambridge, United Kingdom, and approved May 29, 2012 (received for review February 21, 2012)

\begin{abstract}
The availability of stable human antibody reagents would be of considerable advantage for research, diagnostic, and therapeutic applications. Unfortunately, antibody variable heavy and light domains $\left(V_{H}\right.$ and $\left.V_{L}\right)$ that mediate the interaction with antigen have the propensity to aggregate. Increasing their aggregation resistance in a general manner has proven to be a difficult and persistent problem, due to the high level of sequence diversity observed in human variable domains and the requirement to maintain antigen binding. Here we outline such an approach. By using phage display we identified specific positions that clustered in the antigen binding site $\left(28,30-33,35\right.$ in $\mathrm{V}_{\mathrm{H}}$ and 24, 49-53, 56 in $\left.\mathrm{V}_{\mathrm{L}}\right)$. Introduction of aspartate or glutamate at these positions endowed superior biophysical properties (non-aggregating, well-expressed, and heat-refoldable) onto domains derived from common human germline families $\left(V_{H} 3\right.$ and $\left.V_{\kappa} 1\right)$. The effects of the mutations were highly positional and independent of sequence diversity at other positions. Moreover, crystal structures of mutant $V_{H}$ and $V_{L}$ domains revealed a surprising degree of structural conservation, indicating compatibility with $\mathrm{V}_{\mathrm{H}} / \mathrm{V}_{\mathrm{L}}$ pairing and antigen binding. This allowed the retrofitting of existing binders, as highlighted by the development of robust high affinity antibody fragments derived from the breast cancer therapeutic Herceptin. Our results provide a general strategy for the generation of human antibody variable domains with increased aggregation resistance.
\end{abstract}

biotechnology | monoclonal antibodies | protein aggregation | protein engineering | antibody therapeutics

Drote rotein aggregation represents a key bottleneck in the generation of antibody-based reagents and hinders the development and production of human therapeutics (1). It is generally believed that the aggregation propensity of larger antibody reagents (such as immunoglobulin $\mathrm{G}$ and $\mathrm{Fab}$ ) is mostly determined by their variable domain components $\left(\mathrm{V}_{\mathrm{H}}\right.$ and $\left.\mathrm{V}_{\mathrm{L}}\right)$, although there is currently little understanding of the mechanisms involved (1, 2). Indeed, significant differences of aggregation rates have been reported for antibodies that differ exclusively in their variable domains $(1,2)$. Aggregation propensity is even more pronounced for smaller antibody reagents, which lack the interdomain stabilization of their larger counterparts $(3,4)$. This is a major problem in biotechnology due to an increasing trend toward smaller antibody formats for imaging and tumor targeting applications (4). Common formats include human single chain fragments (scFv) and human single domain antibodies, both of which frequently display poor biophysical properties $(1,3)$.

The aggregation propensity of human variable domains is in marked contrast to the variable heavy domains of camels and llamas, which are generally nonaggregating and soluble (" $\mathrm{V}_{\mathrm{HH}}$ domains") (5-7). More favorable properties have also been described for "camelized" and other engineered human $V_{H}$ model domains (8-12) but not for human $\mathrm{V}_{\mathrm{L}}$ domains. Their properties have been attributed to extensive structural changes resulting in increased hydrophilicity of the $\mathrm{V}_{\mathrm{H}}-\mathrm{V}_{\mathrm{L}}$ interface $(8,10,12)$.

However, the relevance of observations in model $\mathrm{V}_{\mathrm{H}}$ domains for the development of antibody reagents for real-world applications had so far remained unclear. In particular, high-affinity antigen binding has not been demonstrated $(8,11)$. Neither has pairing of the model domains with human $\mathrm{V}_{\mathrm{L}}$, a property also observed for camelid domains, which are naturally devoid of light chain partners (and for which analogous structural features in the former $\mathrm{V}_{\mathrm{H}}-\mathrm{V}_{\mathrm{L}}$ interface have been reported) $(8,10-12)$. Moreover, human antibody variable domains are highly diverse and encompass multiple germline families for both heavy and light domains $(13,14)$. Even among variable domains derived from a single germline family considerable diversity is observed, predominantly within complementarity determining regions (CDR) (13-15). The high level of diversity affects aggregation propensity and biophysical properties of human variable domains. For instance, it has been demonstrated that both the choice of germline family and the composition of CDR3 have major influences on aggregation propensity (3). This is supported by reports from our laboratory indicating that preselection for aggregation-resistant $\mathrm{V}_{\mathrm{H}}$ domains results in a reduction of CDR diversity by several orders of magnitude (16-18). As CDR3 mediates the majority of contacts with antigen (19), the question whether high affinity antigen binding and aggregation resistance are in fact mutually compatible had so far remained unanswered.

In this study we outline a general strategy for the generation of human antibody variable domains with increased aggregation resistance. We demonstrate its applicability to common human variable domain families and diverse antibody repertoires and present a report of human $\mathrm{V}_{\mathrm{L}}$ domains with improved biophysical properties. Moreover, we present structural and mutational evidence demonstrating compatibility with high-affinity antigen binding.

\section{Results}

Phage Display Screen for Aggregation Preventing Mutations in Human Antibody Variable Domains. We utilized a high-throughput method

Author contributions: K.D., R.R., D.L., D.S., and D.C. designed research; K.D., R.R., I.K., P.S., J.S., D.L., and D.C. performed research; K.D., R.R., D.L., D.S., and D.C. analyzed data; and D.S. and D.C. wrote the paper.

Conflict of interest statement: K.D., R.R., and D.C. are named inventors on patents relating to this work and declare competing financial interests.

This article is a PNAS Direct Submission.

Data deposition: The atomic coordinates and structure factors have been deposited in the Protein Data Bank, www.pdb.org (PDB ID code 3UPC and 3UPA).

${ }^{1}$ K.D. and R.R. contributed equally to this work.

${ }^{2}$ To whom correspondence should be addressed. E-mail: d.christ@garvan.org.au.

This article contains supporting information online at www.pnas.org/lookup/suppl/ doi:10.1073/pnas.1202866109//DCSupplemental. 
on phage (20) to identify mutations that render human antibody variable domains resistant to aggregation. For our studies we chose variable domains that belong to families that are among the most common in the human repertoire $\left(\mathrm{V}_{\mathrm{H}} 3, \mathrm{~V}_{\kappa} 1\right)(13,14)$. These variable domain families are also prevalent among the 26 human monoclonal antibodies in clinical use and more than 350 currently in clinical trials $(4,21)$. Human $\mathrm{V}_{\mathrm{H}}$ and $\mathrm{V}_{\mathrm{L}}$ domains with amino acid substitutions at surface-exposed positions were displayed on phage. We targeted a wide range of positions while excluding CDR3 to limit effects on antigen binding. The domains were then heated and captured using protein A or L superantigens (which bind to folded but not to aggregated variable domains) $(20,22)$. Wild-type domains readily aggregated under such conditions $\left(80^{\circ} \mathrm{C}\right)$. However, this was not observed for several of the mutant domains, for which superantigen binding after heating was considerably improved (up to 40-fold for human $\mathrm{V}_{\mathrm{H}}$ and 80 -fold for human $V_{L}$ ) (Fig. 1 and Fig. S1). While we observed improvements when making both positively and negatively charged substitutions, by far the largest effect was observed for negatively charged substitutions (glutamate and, in particular, aspartate) (Fig. S2).

Mutations in the Antigen-Binding Site Control Aggregation Propensity of Common Human Variable Domain Families. Moreover, our experiments revealed that these effects were highly positional, highlighting specific sites that control aggregation resistance of the domains. In the case of human $\mathrm{V}_{\mathrm{H}}$ such mutations clustered in CDR1 (H1), while mutations at other positions had little effect. Intriguingly, we discovered that this was not the case for human $\mathrm{V}_{\mathrm{L}}$ : Here mutations strongly clustered in CDR2 (L2) (Fig. $1 A$ and $B)$. In total we identified six positions in $\mathrm{H} 1(28,30,31,32$,
$33,35)$ and five positions in $\mathrm{L} 2(50,51,52,53,56)$ that had major effects on aggregation resistance (in addition to two non-CDR positions at 24 and 49 of $\mathrm{V}_{\mathrm{L}}$ ). In addition to highlighting the positional nature of mutations, our experiments also demonstrated that their effects were largely additive, with combinations of mutations providing the highest levels of aggregation resistance (Fig. $1 C$ ). To demonstrate that our findings were more generally applicable, we next investigated the effect of mutations in $\mathrm{H} 1$ and L2 on antibody repertoires. For this purpose, we constructed synthetic phage display repertoires mimicking the CDR amino acid diversity in the human $\mathrm{V}_{\mathrm{H}}\left(\mathrm{V}_{\mathrm{H}} 3\right)$ and $\mathrm{V}_{\mathrm{L}}\left(\mathrm{V}_{\kappa} 1\right)$ repertoire (23). This revealed that the introduction of double aspartate substitutions into $\mathrm{H} 1$ of human $\mathrm{V}_{\mathrm{H}}$ and $\mathrm{L} 2$ of human $\mathrm{V}_{\mathrm{L}}$ significantly increased the mean aggregation resistance of the repertoires $(p<0.001)$ (Fig. 1D). We observed a dominant effect of mutations in $\mathrm{H} 1$ and L2: Thus, the observed aggregation resistance was largely independent of diversity at other CDR positions.

Human Variable Domains with Superior Biophysical Properties. Representative human variable domains were next expressed as soluble proteins to further assess their biophysical properties. As previously reported, human variable domains rapidly aggregate when heated to $80-85^{\circ} \mathrm{C}$, conditions well above their melting temperatures (20). In addition, they have a tendency to "stick" to gel filtration matrices, which hinders purification, and manifests itself through increased elution volumes (3, 20). Many human variable domains are also poorly expressed $(3,20)$. We observed that introduction of negatively charged substitutions (aspartate or glutamate) at $\mathrm{H} 1$ and $\mathrm{L} 2$ positions improved on all of the above properties. While unmodified human variable domains rapidly aggregated when subjected to heating, the mutant domains
A

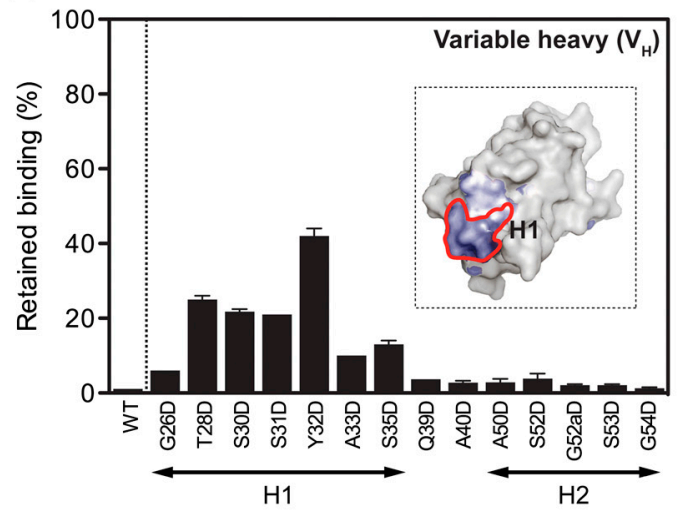

C
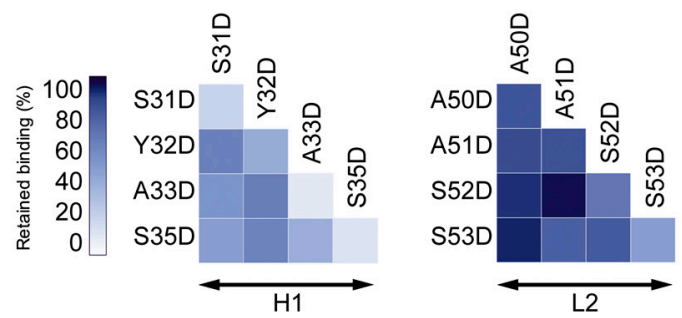

B

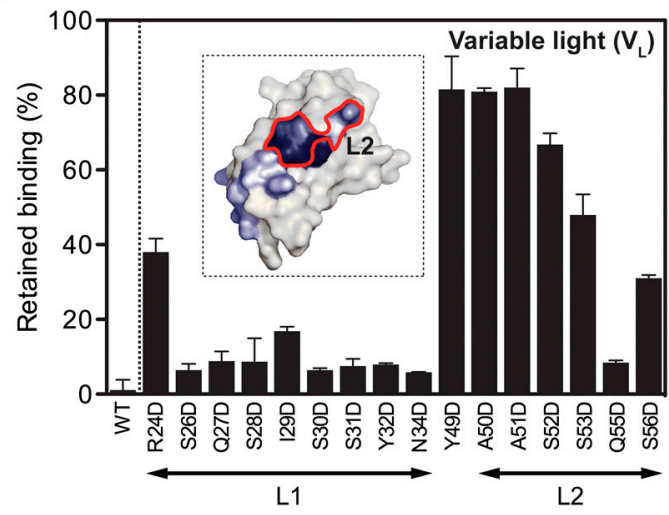

D

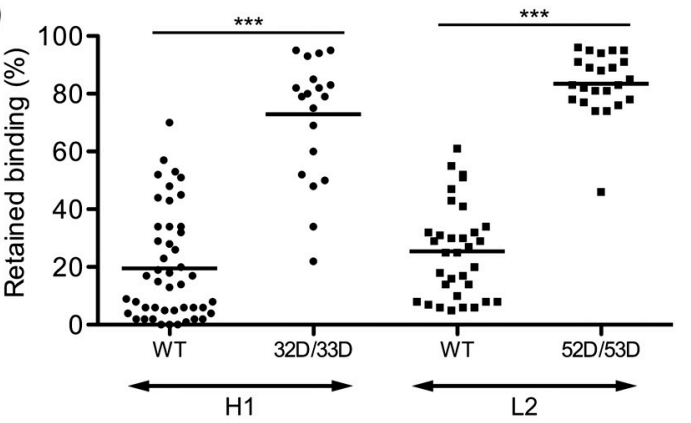

Fig. 1. Effect of mutations in human antibody variable domains on aggregation resistance. Surface residues in variable heavy and light domains (human $\mathrm{V}_{\mathrm{H}} 3$, human $\mathrm{V}_{\kappa} 1$ ) are targeted for substitution with aspartic acid (aspartate). Aggregation resistance of the domains is determined by measuring retained binding to superantigen after heating to $80^{\circ} \mathrm{C}$ on phage (20). Mutations are mapped on the variable domain surface structure (blue: $100 \%$ retained binding; white: $0 \%$; wild type residue: WT; means, standard deviation (SD) shown, $n=2)$. Numbering according to Kabat (30). Complementarity determining regions are indicated as $\mathrm{H} 1, \mathrm{H} 2$ for $\mathrm{V}_{\mathrm{H}}$ and $\mathrm{L} 1, \mathrm{~L} 2$ for $\mathrm{V}_{\mathrm{L}}$. (A) Single mutations in human $\mathrm{V}_{\mathrm{H}}$. (B) Single mutations in human $\mathrm{V}_{\mathrm{L}}$. (C) Double mutations in human $\mathrm{V}_{\mathrm{H}}\left(\right.$ Left) and human $\mathrm{V}_{\mathrm{L}}$ (Right). (D) Mutations in human $\mathrm{V}_{\mathrm{H}}$ repertoire (Left) and human $\mathrm{V}_{\mathrm{L}}$ repertoire (Right). Synthetic repertoires closely mimicking CDR amino acid diversity in the natural antibody repertoire were generated (23). Mutant repertoires carry mutations in H1 (32D/33D) and L2 (52D/53D). Graph shows mean aggregation resistance of repertoires $(* * *, p<0.001)$. 
A

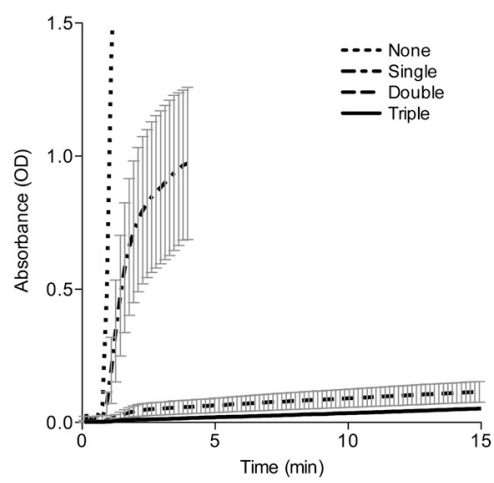

B

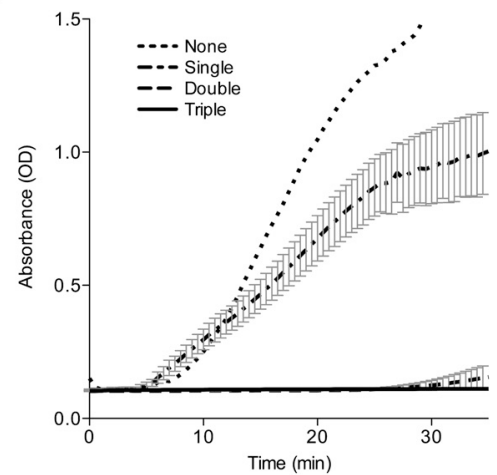

Fig. 2. Aggregation resistance of human variable domains. Representative human variable domains are targeted for substitution with aspartate/glutamate in $\mathrm{CDR} H 1\left(\mathrm{~V}_{\mathrm{H}}\right)$ or $\mathrm{L} 2\left(\mathrm{~V}_{\mathrm{L}}\right)$, expressed and purified (see SI Text for details). The graphs show sample turbidity after incubation at aggregation-promoting temperatures (as measured by absorbance at $360 \mathrm{~nm}$; means, SD shown for single and double mutations, $\mathrm{V}_{\mathrm{H}}: n=3, \mathrm{~V}_{\mathrm{L}}: n=4$ ). $(A)$ Human $\mathrm{V}_{\mathrm{H}}$ domains at $80^{\circ} \mathrm{C}$. (B) Human $\mathrm{V}_{\mathrm{L}}$ domains at $85^{\circ} \mathrm{C}$.

resisted aggregation (Fig. 2). Other biophysical properties of the mutant domains were also greatly improved. This included reduced elution volumes on gel filtration and improved refolding yields (Table 1). The mutant domains also resisted aggregation when subjected to commonly used storage and concentration techniques, including freeze/drying and membrane filtration (Fig. S3). Moreover, soluble expression levels increased by more than $100 \%$ for $V_{L}$ domains and $400 \%$ for $V_{H}$ (Table 1). For all of the observed effects, we noticed a strong dependence on the number of mutations, with two or more mutations providing maximum aggregation resistance.

Structures of Mutant Human $\mathbf{V}_{\mathbf{H}}$ and Human $\mathbf{V}_{\mathbf{L}}$ Domains. To investigate the molecular basis of mutations in CDR H1 and L2 we determined crystal structures of triple mutants of human $\mathrm{V}_{\mathrm{H}}$ and human $\mathrm{V}_{\mathrm{L}}$ domains at $2.8-\AA$ and $1.8-\AA$ resolution (Fig. $3 A$ and $B$ and Fig. S4 and Table S1). The overall structures of the mutant domains closely aligned with structures of variable domains from representative human antibodies (with backbone rmsds of $0.5 \AA$ for $\mathrm{V}_{\mathrm{H}}$ and $0.8 \AA$ for $\mathrm{V}_{\mathrm{L}}$ ). Moreover, no structural changes were observed for either the $\mathrm{V}_{\mathrm{H}}-\mathrm{V}_{\mathrm{L}}$ interface or mutated CDR regions, with conformations of $\mathrm{H} 1$ and L2 tightly superimposing onto the representative antibody variable domain structures (Fig. $3 \mathrm{~A}$ and $B)$. Our results demonstrated that structural changes in the mutant variable domains were minor and exclusively limited to mutant side

Table 1. Biophysical properties of human antibody variable domains

\begin{tabular}{lrrrr} 
& \multicolumn{4}{c}{ Mutations } \\
& None & Single & Double & Triple \\
\hline Expression (mg/L) & & & & \\
$V_{H}$ & 1.7 & 4.7 & 13.3 & 11.0 \\
$V_{L}$ & 47.0 & 78.6 & 103.7 & 104.6 \\
Elution volume (mL) & & & & \\
$V_{H}$ & 24.9 & 23.1 & 21.3 & 19.7 \\
$V_{L}$ & 13.6 & 13.3 & 13.0 & 12.9 \\
Heat refolding (\%) & & & & \\
$V_{H}$ & 4.0 & 69.3 & 82.3 & 88.0 \\
$V_{L}$ & 53.6 & 72.5 & 67.1 & 91.4 \\
\hline
\end{tabular}

Representative human variable domains are targeted for substitution with aspartate/glutamate in CDR H1 $\left(\mathrm{V}_{\mathrm{H}}\right)$ or L2 $\left(\mathrm{V}_{\mathrm{L}}\right)$, expressed and purified (see $S /$ Text for details) (means shown for single and double mutations, $\left.\mathrm{V}_{\mathrm{H}}: n=3, \mathrm{~V}_{\mathrm{L}}: n=4\right)$ ). Expression levels are determined by superantigen capture from culture supernatant. Elution volumes are determined by size-exclusion chromatography. For heat refolding, samples are incubated for $10 \mathrm{~min}$ at $80^{\circ} \mathrm{C}\left(\mathrm{V}_{\mathrm{H}}\right)$ or $20 \mathrm{~min}$ at $85^{\circ} \mathrm{C}\left(\mathrm{V}_{\mathrm{L}}\right)$ and analyzed by size-exclusion chromatography. chains. The absence of conformational rearrangements also raised the possibility that the mutations may be compatible with $\mathrm{V}_{\mathrm{H}}-\mathrm{V}_{\mathrm{L}}$ pairing and antigen binding when introduced into an existing monoclonal.

Retrofitting of Variable Domains. To further investigate the effect of the identified mutations on the antibody-antigen interaction, we decided to study variants of Trastuzumab (Herceptin), an IgG therapeutic monoclonal antibody directed against human epidermal growth factor receptor 2 (HER2) (24). Single and double aspartate substitutions were introduced into $\mathrm{H} 1$ and/or L2 and variants expressed in a human IgG1 format (see SI Text for details). We then analyzed the effects of the substitutions on biological activity. Experiments using the HER2-expressing breast cancer cell line SK-BR-3 revealed that the variants were highly active, with only minor differences in cellular binding and inhibition of proliferation observed (Fig. $4 A$ and $B$ ). Furthermore, no differences were detected for serum clearance when injected into animals at doses relevant to human therapy (Fig. 4C). We next investigated if $\mathrm{H} 1$ and/or L2 mutations were also compatible with antigen binding in an antibody fragment format. While isolated variable domains displayed little binding to antigen, binding activity could be reconstituted by pairing the domains in an $\mathrm{scFv}$ format (Fig. S5). This allowed us to measure the binding of scFv variants for recombinant HER2 by surface plasmon resonance (Fig. 5A). Some of the variants lost much of their affinity for HER2; these generally carried mutations at positions reported to directly interact with antigen (25). However, changes at other positions were well tolerated, with no apparent loss of equilibrium binding affinity $\left(\mathrm{K}_{\mathrm{D}}\right)$. Moreover, no loss of binding was observed when combining single changes at tolerated H1 and L2 positions. In particular, one of the scFv double mutants $(30 \mathrm{D} /$ 52D) bound to HER2 with wild-type-like affinity (Fig. 5A).

To examine resistance against aggregation, 30D/52D and other variants were next heated to $80-85^{\circ} \mathrm{C}$ at high protein concentration $(10-100 \mu \mathrm{M})$. As we had observed for germline variable domains, aggregation resistance improved considerably as the number of mutations increased: While the wild-type scFv fragment readily aggregated at the above conditions, the $30 \mathrm{D} / 52 \mathrm{D}$ double mutant resisted aggregation, with single mutations providing intermediate effects (Fig. 5B). Indeed, simple visual inspection of protein solutions after heating readily revealed considerable improvements of aggregation resistance for single domains as well as when combined in an $\mathrm{scFv}$ format (Fig. $5 C$ ).

\section{Discussion}

Taken together, our experiments demonstrate that aggregation resistant human antibody variable domains can be generated through introduction of charge in the antigen binding site. Previous studies 
A

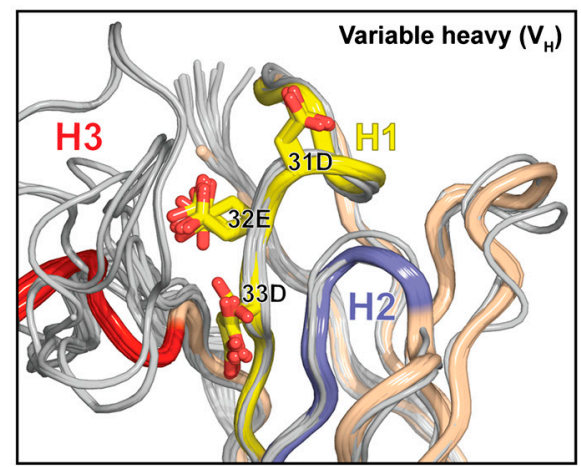

B

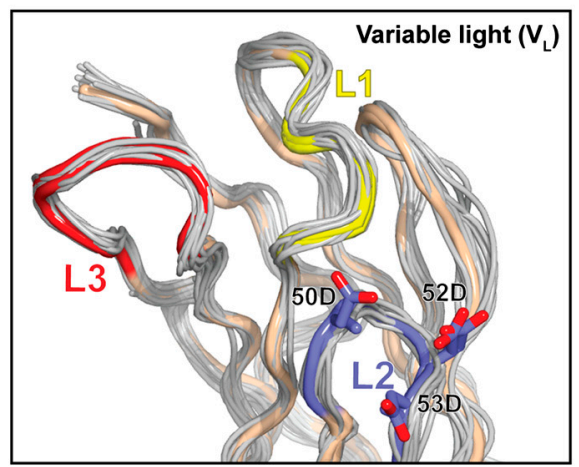

Fig. 3. Crystal structures of mutant human variable domains: structure of triple mutants of $(A)$ human $V_{H}$ and $(B)$ human $V_{L}$ (in tan). CDR regions are shown in yellow/blue/red. Mutant residues are highlighted (sticks). The structures of the mutant domains tightly superpose onto structures of representative human variable domains [with the exception of H3 which is conformationally diverse in antibodies (40); representative structures shown in gray].

had indicated that the introduction of a large number (30-80) of charged substitutions at nonspecific surface positions can generate robust "supercharged" proteins (such as supercharged green fluorescent protein) (26). In marked contrast, the approach described here requires the introduction of only a handful of substitutions (two or more) at highly specific positions $(28,30,31,32,33,35$ in $\mathrm{V}_{\mathrm{H}}$ and $24,49,50,51,52,53,56$ in $\mathrm{V}_{\mathrm{L}}$ ). Unlike supercharged proteins, many of the aggregation-resistant mutant variable domains carry no global net charge, highlighting the importance of localized charge (Fig. S4). We also observe a detectable preference for aspartate over glutamate and a strong preference for aspartate over lysine or arginine (Fig. S2). This indicates that properties other than charge also influence the observed aggregation resistance. While detailed mechanisms remain unclear, a preference for small negatively charged amino acids is apparent. The positional nature of our approach is further highlighted by the differences observed for human $\mathrm{V}_{\mathrm{H}}$ and $\mathrm{V}_{\mathrm{L}}$ with mutations clustering in CDR1 (H1) and CDR2 (L2), respectively (Fig. $1 A$ and $B$ ). In contrast to human $\mathrm{V}_{\mathrm{H}}$, mutations in CDR1 have little effect on human $\mathrm{V}_{\mathrm{L}}$ despite the fact that the domains are structurally closely related immunoglobulin folds.

The mutations reported here endow aggregation resistance onto domains derived from common germline families $(13,14)$, with $\mathrm{V}_{\mathrm{H}} 3$ and $\mathrm{V}_{\kappa} 1$ representing approximately $40 \%$ and $32 \%$ of the rearranged repertoire (23). Importantly, we find that the effects of mutations in CDR H1 and L2 are not significantly influenced by diversity at other CDR positions (Fig. 1D). This is despite the fact that the expressed human variable domain repertoire is highly diverse (15). This is reflected by the synthetic repertoires studied here, which are extensively randomized at between $9\left(\mathrm{~V}_{\kappa} 1\right)$ and $14 \mathrm{CDR}$ positions $\left(\mathrm{V}_{\mathrm{H}} 3\right)$. The synthetic variable domain repertories are designed to reflect natural sequence diversity within the human $\mathrm{V}_{\kappa} 1$ and $\mathrm{V}_{\mathrm{H}} 3$ repertoire. Thus, we utilize preferentially rearranged human gene segments (V3-23/ DP47, O12/O2/DPK9) $(27,28)$ and use trinucleotide phosphoramidite mutagenesis to match CDR amino acid distributions (29) (see SI Text for details). An alternative approach to repertoire diversity relies on the use of "consensus" sequences (rather than single common gene segments) (23). This strategy was used in the development of the Herceptin $\left(\mathrm{V}_{\mathrm{H}} 3\right.$ and $\left.\mathrm{V}_{\kappa} 1\right)$ variable domains (24) and is also clearly compatible with the mutational strategy outlined here (Fig. 3).

It is important to note that multiple mutations (two or more) are required to obtain considerable improvements of aggregation resistance in common biophysical assays (Fig. 2). The requirement for multiple positional substitutions may explain why effects on commercially important and widely studied immunoglobulin families had so far remained unnoticed. It also renders it unlikely that such mutations could be observed by chance within the natural repertoire. Thus, multiple aspartates or glutamates are not common at identified positions within the human $\mathrm{V}_{\mathrm{H}} 3$ and $\mathrm{V}_{\kappa} 1$ germline repertoire and are notably absent from frequently utilized gene segments (such as V3-23) $(27,28)$. They are also not common in rearranged human antibody sequences (30), although exceptions exist [such as the Adalimumab (Humira) $\mathrm{V}_{\mathrm{H}}$ domain] (31).

Our approach provides favorable characteristics among a range of biophysical properties. This includes considerable increases in expression yields, improved concentration, and purification. It also endows human variable domains with the capability
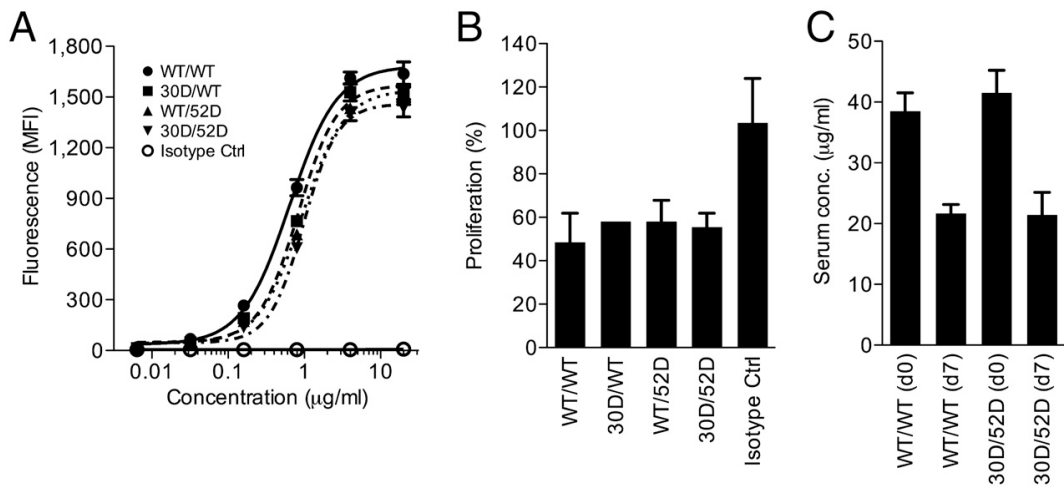

Fig. 4. Retrofitting of human variable domains (I): effects on biological activity of IgG. Variants of Trastuzumab (Herceptin) are generated by introduction of aspartate substitutions in CDR H1 and/or L2. Means, SD shown. (A) Binding to HER2 antigen on cells. The graph shows binding of IgG to SK-BR-3 breast cancer cells $(n=2)$. (B) Inhibition of cellular proliferation (as determined by incubation of SK-BR-3 cells with IgG; $n=2$ ). (C) Serum concentrations (after intra-peritoneal injection of IgG into C57/BL6 mice at $1 \mathrm{mg} / \mathrm{kg}, n=4)$. 
A

\begin{tabular}{lllll}
\hline \multicolumn{3}{c}{ Mutations } & \multicolumn{3}{c}{ HER2 affinity } \\
\hline $\mathbf{H 1}$ & $\mathbf{L 2}$ & $\mathbf{k}_{\mathrm{a}}\left(10^{5} \mathrm{M}^{-1} \mathrm{~s}^{-1}\right)$ & $\mathbf{k}_{\mathrm{d}}\left(10^{-4} \mathrm{~s}^{-1}\right)$ & $\mathrm{K}_{\mathrm{D}}(\mathrm{nM})$ \\
\hline- & - & 1.3 & 4.9 & 3.8 \\
\hline 30D & - & 2.4 & 4.9 & 2.3 \\
- & 52D & 1.2 & 9.1 & 7.6 \\
- & 53D & 1.5 & 175 & 118 \\
\hline 30D & 52D & 2.1 & 8.6 & 4.1 \\
\hline 30D & 52D-53D & 0.4 & 192 & 472 \\
\hline
\end{tabular}

B

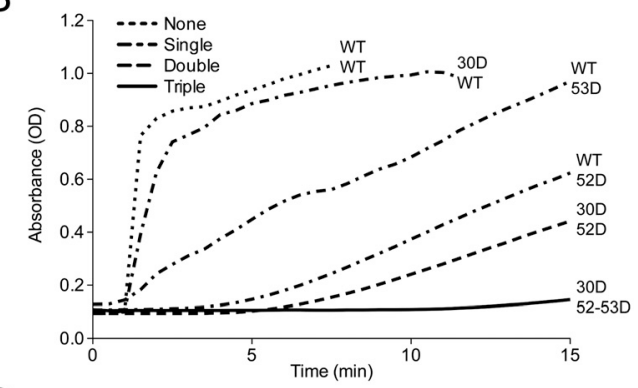

C

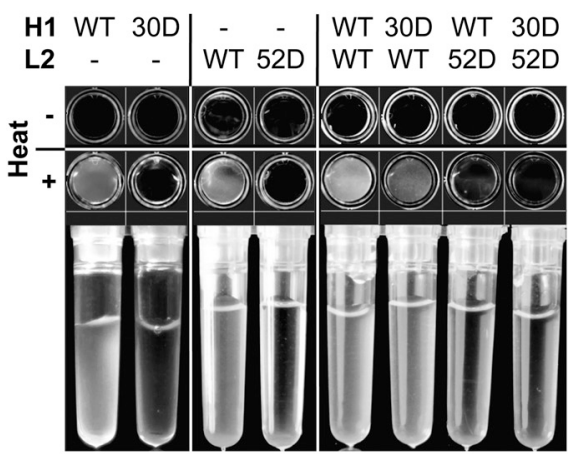

Fig. 5. Retrofitting of human variable domains (II): effects on antibody fragments. Variants of Trastuzumab (Herceptin) are generated by introduction of aspartate substitutions in CDR H1 and/or L2. (A) Affinity of mutants for recombinant HER2 (as determined by surface plasmon resonance using scFv fragments). (B) Aggregation resistance of mutants. The graph shows sample turbidity of scFv fragments at $85^{\circ} \mathrm{C}$ (as measured by absorbance at $360 \mathrm{~nm}$ ). (C) Aggregation resistance (visual appearance) before (-) and after ( + ) heating to $85^{\circ} \mathrm{C}$. Panel shows $\mathrm{V}_{\mathrm{H}}$ domains (Left), $\mathrm{V}_{\mathrm{L}}$ domains (Center), and paired as SCFv fragments (Right).

to refold after heat-induced unfolding. In this respect, the mutant domains closely resemble camelid $\left(\mathrm{V}_{\mathrm{HH}}\right)$ variable domains, which display this otherwise uncommon behavior (5). Differences in CDR conformation and the structure of the $\mathrm{V}_{\mathrm{H}^{-}}-\mathrm{V}_{\mathrm{L}}$ interface have been shown to underpin the observed biophysical differences between human $\mathrm{V}_{\mathrm{H}}$ and camelid domains (7). Similar features have been described for camelized human $\mathrm{V}_{\mathrm{H}}$ domains carrying nonhuman framework mutations and other human model $\mathrm{V}_{\mathrm{H}}$ domains (such as HEL4) $(8,12) . \mathrm{V}_{\mathrm{HH}} \mathrm{S}$ also generally display higher thermodynamic stabilities (6); however, this is not observed for the mutant human domains (Table S2). The crystal structures of the mutant human domains reported here further highlight differences with $\mathrm{V}_{\mathrm{HH}}$, with the domains fully retaining human CDR conformations. Previously reported structural features of human model $\mathrm{V}_{\mathrm{H}}$ domains centered on hydrophobic framework residues and rearrangement of the $\mathrm{V}_{\mathrm{H}}-\mathrm{V}_{\mathrm{L}}$ interface are also noticeably absent from the structures (Fig. S7) (7, 8, 10-12, 32).

The observation that the identified mutations in human variable domains result in neither structural change nor increased thermodynamic stability indicates that these parameters are unlikely to be a cause for the observed aggregation resistance. However, it should be noted that the variable domains utilized in this study belong to families $\left(\mathrm{V}_{\mathrm{H}} 3, \mathrm{~V}_{\kappa} 1\right)$ that are not only among the most common but also among the more stable within the human repertoire (but less stable than camelid domains) $(3,6)$. It does not exclude the possibility that some of the less stable human families may require additional stabilization (such as $\left.\mathrm{V}_{\mathrm{H}} 2\right)(3,32)$. In contrast, the mutant variable domains described in this study are actually moderately less stable than the domains from which they were derived (Table S2; $\Delta G_{N-U}: 27-40 \mathrm{~kJ} \mathrm{~mol}^{-1}$ for $\mathrm{V}_{\mathrm{H}}$ and 24-30 kJ mol${ }^{-1}$ for $V_{L}$ ). This is in agreement with observations reported for model $\mathrm{V}_{\mathrm{H}}$ domains $(10,11)$ as well as for other proteins (33). Indeed, it has been suggested that mutations that reduce protein aggregation frequently do not increase thermodynamic stability but rather act on aggregation-prone unfolded or partially unfolded states $(20,33)$.

The absence of structural changes in human $V_{H}$ and human $V_{L}$ also suggests that the identified mutations are likely to be compatible with $\mathrm{V}_{\mathrm{H}}-\mathrm{V}_{\mathrm{L}}$ pairing and antigen binding when introduced into existing binders. It does not exclude the possibility of direct disruption of side-chain interactions with antigen, which has to be empirically determined for each binder. However, the positions highlighted in this study fall predominantly into CDR H1 and L2 regions, which are mostly peripheral to the antibody-antigen interaction, rendering such disruptions less likely (15). This is in contrast to CDR H3 and L3, which mediate the majority of interactions with antigen $(19,34)$. It should also be noted that the approach outlined here provides a considerable degree of flexibility because it identified a large set of positions in both human chains ( six in $\mathrm{V}_{\mathrm{H}}$ and seven in $\mathrm{V}_{\mathrm{L}}$ ). Both aspartate and glutamate can be introduced at these positions, although we observe a detectable preference for the former (Fig. S2). In addition, the required number of changes is modest, with two or more mutations providing a high degree of aggregation resistance (Fig. 2). The broad applicability of our approach is further exemplified by the work on Herceptin variants for which tolerated mutations in CDR $\mathrm{H} 1 / \mathrm{L} 2$ could be readily identified (Figs. 4 and 5). This is not unexpected because it has been demonstrated that the HerceptinHER2 interaction is dominated by a small number of residues providing large contributions to binding energy (35). These residues cluster predominantly in CDR H3/L3 (35) and contact the C-terminal portion of domain IV of HER2 (36).

While we were preparing this paper, two publications have appeared in print that further highlight the role of charge in antibody aggregation. Perchiacca et al. describe a set of mutants of the model $\mathrm{V}_{\mathrm{H}}$ HEL4 (11). In particular, they report effects of a "triad" of negatively charged surface residues in HEL4 (but do not attribute effects to single or double mutations). This triad has previously been described (9) and is in agreement with the results outlined here. The authors do not provide evidence on antigen binding, repertoire diversity, or $\mathrm{V}_{\mathrm{H}}-\mathrm{V}_{\mathrm{L}}$ pairing, making their findings difficult to assess. Schaefer and Pluckthun outline a strategy of fusing a negatively charged peptide to the $\mathrm{N}$ terminus of human antibodies to increase aggregation resistance (37). While their approach is clearly compatible with antigen binding, the observed effect is not positional and can be attributed to increased net charge of the molecule (37). It may, however, provide synergies with the strategy outlined here.

Although the role of mutations in the antigen binding site on aggregation propensity had so far remained elusive, the effect of such mutations on other antibody properties are well understood. In particular, it has been demonstrated that the binding activity of one antibody can be transferred onto a different antibody exclusively through mutations in the antigen-binding site ("CDR grafting") $(24,34)$. The majority of monoclonal antibodies currently in clinical practice have been generated by CDR grafting approaches (38). Other monoclonals have been generated through the introduction of synthetic, oligonucleotide-derived diversity in CDR regions (38). An initial concern relating to CDR grafting/mutagenesis strategies, including the approach outlined here, had been the generation of potential new B- and T-cell epitopes (39). However, with a large number of monoclonals in 
clinical practice it has become evident that CDR mutations cause little or no immunogenicity in humans (39). In contrast, there is increasing evidence that the absence of aggregates is essential for the development of antibody therapeutics with low immunogenicity $(1,2)$. The method outlined here is therefore highly compatible with current antibody development strategies. This is exemplified by our work on variants of the antibody therapeutic Herceptin and highlights the potential of our method for "retrofitting" approaches.

\section{Conclusion}

The availability of antibody reagents with improved aggregation resistance would be a significant advantage for research, diagnostic, and therapeutic applications. Consequently, the discovery of stable and nonaggregating $\mathrm{V}_{\mathrm{H}}$ domains from camels and llamas has generated considerable interest and development efforts in recent years $(4,5)$. While such domains hold promise as robust and modular building blocks, their lack of light-chain partners

1. Lowe D, et al. (2011) Aggregation, stability, and formulation of human antibody therapeutics. Adv Protein Chem Struct Biol 84:41-61.

2. Demarest SJ, Glaser SM (2008) Antibody therapeutics, antibody engineering, and the merits of protein stability. Curr Opin Drug Discov Devel 11:675-687.

3. Ewert S, Huber T, Honegger A, Pluckthun A (2003) Biophysical properties of human antibody variable domains. $J \mathrm{Mol} \mathrm{Biol} 325: 531-553$.

4. Holliger P, Hudson PJ (2005) Engineered antibody fragments and the rise of single domains. Nat Biotechnol 23:1126-1136.

5. Hamers-Casterman C, et al. (1993) Naturally occurring antibodies devoid of light chains. Nature 363:446-448.

6. Ewert S, Cambillau C, Conrath K, Pluckthun A (2002) Biophysical properties of camelid $\mathrm{V}(\mathrm{HH})$ domains compared to those of human $\mathrm{V}(\mathrm{H}) 3$ domains. Biochemistry 41:3628-3636.

7. Muyldermans S, Atarhouch T, Saldanha J, Barbosa JA, Hamers R (1994) Sequence and structure of $\mathrm{VH}$ domain from naturally occurring camel heavy chain immunoglobulins lacking light chains. Protein Eng 7:1129-1135.

8. Barthelemy PA, et al. (2008) Comprehensive analysis of the factors contributing to the stability and solubility of autonomous human VH domains. I Biol Chem 283:3639-3654.

9. Christ D, Dudgeon K (2011) Modified Variable Domain Molecules and Methods for Producing and Using Same. WO/2011/047442 (April 04, 2011).

10. Jespers L, Schon O, James LC, Veprintsev D, Winter G (2004) Crystal structure of HELC a soluble, refoldable human $\mathrm{V}(\mathrm{H})$ single domain with a germ-line scaffold. J Mol Biol 337:893-903.

11. Perchiacca JM, Bhattacharya M, Tessier PM (2011) Mutational analysis of domain antibodies reveals aggregation hotspots within and near the complementarity determin ing regions. Proteins 79:2637-2647.

12. Riechmann $L$ (1996) Rearrangement of the former $V L$ interface in the solution structure of a camelised, single antibody VH domain. J Mol Biol 259:957-969.

13. Cox JP, Tomlinson IM, Winter G (1994) A directory of human germ-line V kappa segments reveals a strong bias in their usage. Eur J Immunol 24:827-836.

14. Tomlinson IM, Walter G, Marks JD, Llewelyn MB, Winter G (1992) The repertoire of human germline $\mathrm{VH}$ sequences reveals about fifty groups of $\mathrm{VH}$ segments with different hypervariable loops. J Mol Biol 227:776-798.

15. Tomlinson IM, et al. (1996) The imprint of somatic hypermutation on the repertoire of human germline $\mathrm{V}$ genes. J Mol Biol 256:813-817.

16. Christ D, Famm K, Winter G (2006) Tapping diversity lost in transformations-In vitro amplification of ligation reactions. Nucleic Acids Res 34:e108.

17. Christ D, Famm K, Winter G (2007) Repertoires of aggregation-resistant human antibody domains. Protein Eng Des Sel 20:413-416

18. Dudgeon K, Famm K, Christ D (2009) Sequence determinants of protein aggregation in human VH domains. Protein Eng Des Sel 22:217-220.

19. MacCallum RM, Martin AC, Thornton JM (1996) Antibody-antigen interactions: Contact analysis and binding site topography. J Mol Biol 262:732-745.

20. Jespers L, Schon O, Famm K, Winter G (2004) Aggregation-resistant domain antibodies selected on phage by heat denaturation. Nat Biotechnol 22:1161-1165. hinders the generation of multidomain antibody reagents; their nonhuman nature also complicates their use as therapeutics. In contrast, a general means for the development of robust human antibody domains had so far remained elusive. We conclude that our approach provides such a means for the generation of human antibody variable domains with increased aggregation resistance.

\section{Methods}

Generation of mutant variable domains and repertoires, analysis of aggregation resistance on phage, protein expression and purification, expression levels, elution volumes, refolding yields and turbidity measurements, crystal growth, structure solution, refinement and analysis, determination of thermodynamic stabilities, affinity measurements, cellular binding, inhibition of proliferation, and serum clearance are described in detail in SI Text.

ACKNOWLEDGMENTS. We thank staff at the Australian Synchrotron MX2 beamline for support and R. Brink, A. Basten, and J. Sprent for advice. This work was supported by the National Health and Medical Research Council and the Australian Research Council.

21. Nelson AL, Dhimolea E, Reichert JM (2010) Development trends for human monoclonal antibody therapeutics. Nat Rev Drug Discov 9:767-774.

22. Jansson B, Uhlen M, Nygren PA (1998) All individual domains of staphylococcal protein A show Fab binding. FEMS Immunol Med Microbiol 20:69-78

23. Knappik A, et al. (2000) Fully synthetic human combinatorial antibody libraries (HuCAL) based on modular consensus frameworks and CDRs randomized with trinucleotides. J Mol Biol 296:57-86

24. Carter P, et al. (1992) Humanization of an anti-p185HER2 antibody for human cancer therapy. Proc Natl Acad Sci USA 89:4285-4289.

25. Bostrom J, et al. (2009) Variants of the antibody herceptin that interact with HER2 and VEGF at the antigen binding site. Science 323:1610-1614

26. Lawrence MS, Phillips KJ, Liu DR (2007) Supercharging proteins can impart unusual resilience. J Am Chem Soc 129:10110-10112.

27. Suzuki I, Pfister L, Glas A, Nottenburg C, Milner EC (1995) Representation of rearranged $\mathrm{VH}$ gene segments in the human adult antibody repertoire. J Immuno 154:3902-3911.

28. Foster SJ, Brezinschek HP, Brezinschek RI, Lipsky PE (1997) Molecular mechanisms and selective influences that shape the kappa gene repertoire of IgM+B cells. J Clin Invest 99:1614-1627.

29. Virnekas B, et al. (1994) Trinucleotide phosphoramidites: Ideal reagents for the synthesis of mixed oligonucleotides for random mutagenesis. Nucleic Acids Re 22:5600-5607.

30. Kabat E, Wu TT, Perry HM, Kay S, Gottesman CF (1992) Sequences of Proteins of Immunological Interest (Diane Publishing, Darby, PA), 5th Ed, p 2719.

31. Wang X, Das TK, Singh SK, Kumar S (2009) Potential aggregation prone regions in biotherapeutics: A survey of commercial monoclonal antibodies. MAbs 1:254-267.

32. Ewert S, Honegger A, Pluckthun A (2003) Structure-based improvement of the biophysical properties of immunoglobulin VH domains with a generalizable approach Biochemistry 42:1517-1528.

33. Chiti F, Stefani M, Taddei N, Ramponi G, Dobson CM (2003) Rationalization of the effects of mutations on peptide and protein aggregation rates. Nature 424:805-808.

34. Jones PT, Dear PH, Foote J, Neuberger MS, Winter G (1986) Replacing the complementarity-determining regions in a human antibody with those from a mouse. Nature 321:522-525

35. Kelley RF, O'Connell MP (1993) Thermodynamic analysis of an antibody functional epitope. Biochemistry 32:6828-6835.

36. Cho HS, et al. (2003) Structure of the extracellular region of HER2 alone and in com plex with the Herceptin Fab. Nature 421:756-760.

37. Schaefer JV, Pluckthun A (2012) Engineering aggregation resistance in IgG by two independent mechanisms: Lessons from comparison of Pichia pastoris and mammalian cell expression. J Mol Biol 417:309-335.

38. Reichert JM, Rosensweig CJ, Faden LB, Dewitz MC (2005) Monoclonal antibody successes in the clinic. Nat Biotechnol 23:1073-1078.

39. Hwang WY, Foote J (2005) Immunogenicity of engineered antibodies. Methods 36:3-10.

40. Chothia C, Lesk AM (1987) Canonical structures for the hypervariable regions of immunoglobulins. J Mol Biol 196:901-917. 